\title{
X-ray laser-induced ablation of lead compounds
}

V. Hájková ${ }^{1}$, L. Juha ${ }^{1 *}$, P. Boháček ${ }^{1}$, T. Burian ${ }^{1}$, J. Chalupský$^{1}$, L. Vyšín ${ }^{1}$, J. Gaudin $^{2}$, P. A. Heimann ${ }^{3}$, S. P. Hau-Riege ${ }^{4}$, M. Jurek ${ }^{5}$, D. Klinger ${ }^{5}$, J. Pelka $^{5}$, R. Sobierajski ${ }^{5}$, J. Krzywinski ${ }^{6}$, M. Messerschmidt ${ }^{6}$, S. P. Moeller ${ }^{6}$, B. Nagler ${ }^{6}$, M. Rowen ${ }^{6}$, W. F. Schlotter ${ }^{6}$, M. L. Swiggers ${ }^{6}$, J. J. Turner 6 , S. M. Vinko ${ }^{7}$, T. Whitcher ${ }^{7}$, J. Wark ${ }^{7}$, M. Matuchová ${ }^{8}$, S. Bajt ${ }^{9}$, H. Chapman ${ }^{9}$, T. Dzelzainis ${ }^{10}$, D. Riley ${ }^{10}$, J. Andreasson ${ }^{11}$, J. Hajdu ${ }^{11}$, B. Iwan ${ }^{11}$, N. Timneanu ${ }^{11}$,

K. Saksl ${ }^{12}$, R. Fäustlin ${ }^{13}$, A. Singer ${ }^{13}$, K. Tiedtke ${ }^{13}$, S. Toleikis ${ }^{13}$, I. Vartaniants ${ }^{13}$, H. Wabnitz ${ }^{13}$

1) Institute of Physics, Academy of Sciences of the Czech Republic, Na Slovance 2, 18221 Prague 8, Czech Republic ("E-mail: juha@fzu.cz)

2) European XFEL, Notkestrasse 85, D-22607 Hamburg, Germany

3) Lawrence Berkeley National Laboratory, 1 Cyclotron Road, CA 94720, USA, 4) Lawrence Livermore National Laboratory, 7000 East Avenue, Livermore, CA 94550, USA, 5) Institute of Physics, Polish Academy of Sciences, Al. Lotników 32/46, PL-02-668 Warsaw, Poland

6) SLAC National Accelerator Laboratory, 2575 Sand Hill Road, Menlo Park, CA 94025, USA 7) Department of Physics, Clarendon Laboratory, University of Oxford, Parks Road, Oxford, OX1 3PU, UK

8) Institute of Chemical Technology, Technická 5, 16628 Praha 6 - Dejvice, Czech Republic

9) Center for Free-Electron Laser Science, Notkestrasse 85, D-22607 Hamburg, Germany

10) Queen's University Belfast, University Road Belfast, BT7 1NN, Northern Ireland, UK

11) Uppsala University, Uppsala, SE-75124 Sweden

12) Institute of Materials Research SAS, Watsonova 47, 04001 Kosice, Slovak Republic

13) Deutsches Elektronen-Synchrotron DESY, Notkestrasse 85, D-22603 Hamburg, Germany

\begin{abstract}
The recent commissioning of a X-ray free-electron laser triggered an extensive research in the area of X-ray ablation of high-Z, high-density materials. Such compounds should be used to shorten an effective attenuation length for obtaining clean ablation imprints required for the focused beam analysis. Compounds of lead $(\mathrm{Z}=82)$ represent the materials of first choice. In this contribution, single-shot ablation thresholds are reported for $\mathrm{PbWO}_{4}$ and $\mathrm{PbI}_{2}$ exposed to ultra-short pulses of extreme ultraviolet radiation and X-rays at FLASH and LCLS facilities, respectively. Interestingly, the threshold reaches only $0.11 \mathrm{~J} / \mathrm{cm}^{2}$ at $1.55 \mathrm{~nm}$ in lead tungstate although a value of $0.4 \mathrm{~J} / \mathrm{cm}^{2}$ is expected according to the wavelength dependence of an attenuation length and the threshold value determined in the XUV spectral region, i.e., 79 $\mathrm{mJ} / \mathrm{cm}^{2}$ at a FEL wavelength of $13.5 \mathrm{~nm}$. Mechanisms of ablation processes are discussed to explain this discrepancy. Lead iodide shows at $1.55 \mathrm{~nm}$ significantly lower ablation threshold than tungstate although an attenuation length of the radiation is in both materials quite the same. Lower thermal and radiation stability of $\mathrm{PbI}_{2}$ is responsible for this finding.
\end{abstract}

Keywords: extreme ultraviolet laser, x-ray laser, free-electron laser, radiation damage, laser ablation, damage thresholds, single-shot damage, focused beam characterization

\section{INTRODUCTION}

In the last decade, new interaction and imaging experiments (see for example [1-3]) were made possible by bringing short-wavelength $(\lambda<100 \mathrm{~nm})$ free-electron lasers (FEL; see for example [4]) into operation and making them ready for users from scientific community. Another milestone was achieved by obtaining the first lasing in the X-ray spectral

Damage to VUV, EUV, and X-ray Optics III, edited by Libor Juha, Saša Bajt, Richard A. London,

Proc. of SPIE Vol. 8077, 807718 · @ 2011 SPIE · CCC code: 0277-786X/11/\$18 · doi: 10.1117/12.890134

Proc. of SPIE Vol. 8077 807718-1 
region at the LCLS facility [5,6] (Linac Coherent Light Source; Menlo Park, CA) in April 2009. Two more X-ray freeelectron lasers are currently either in commissioning (Spring-8 Angstrom Compact free electron LAser - SACLA in Japan [7]) or under construction (European XFEL in Germany [8]). So, there is an urgent need to modify techniques and instrumentation developed for FELs working in the extreme ultraviolet and the soft X-ray spectral regions for the purpose of their use at X-ray FEL facilities.

At extreme ultraviolet and soft X-ray laser facilities, an ablation of suitable materials has been utilized for visualization and characterization of both lateral and longitudinal distribution of radiation intensity in the focused laser beam [9-11]. Choosing the material most suitable for this purpose, we should take into account an assumption of a local action of deposited energy on ablating material. Materials ablated by short-wavelength radiation in a non-thermal manner, without massive melting and influence of other unwanted phase transitions, represent the best choice. For the wavelengths longer than $10 \mathrm{~nm}$, aliphatic organic polymers, e.g., poly(methyl methacrylate) - PMMA, have been proven as material serving to this purpose very well. Moreover, PMMA is also widely used in electron-beam, EUV, and x-ray lithography as a resist so that its radiation-chemical and radiation-physical properties are very well understood.

However, detailed investigation of the wavelength dependence of PMMA ablation characteristics at the FLASH facility (for more details about the facility, its beamlines and focusing systems see $[12,13]$ ) revealed an increase of surface roughness with decreasing wavelength (Fig. 1). Using X-rays to ablate the polymer, the roughness, resulting from increasing attenuation length of radiation and kinetic energy of photoelectrons, of crater surface make determination of the beam characteristics from crater contours, areas and shapes very difficult and unreliable. AFM image of such a rough surface obtained at LCLS can be seen in Fig. 2. Therefore we should abandon materials like PMMA when we are working at X-ray FEL facilities, turning to heavy solids securing short attenuation length of X-rays.

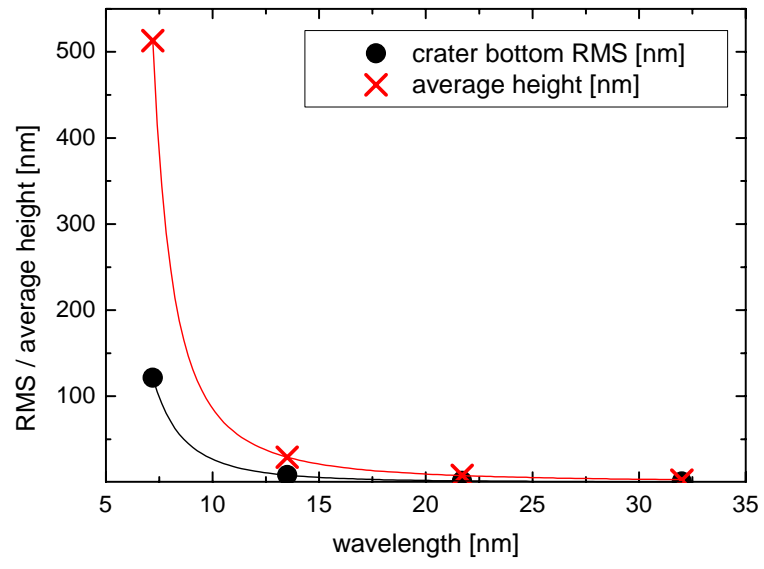

Figure 1: Wavelength dependence of a roughness of PMMA ablated at FLASH tuned from $32 \mathrm{~nm}$ to $7.1 \mathrm{~nm}$.

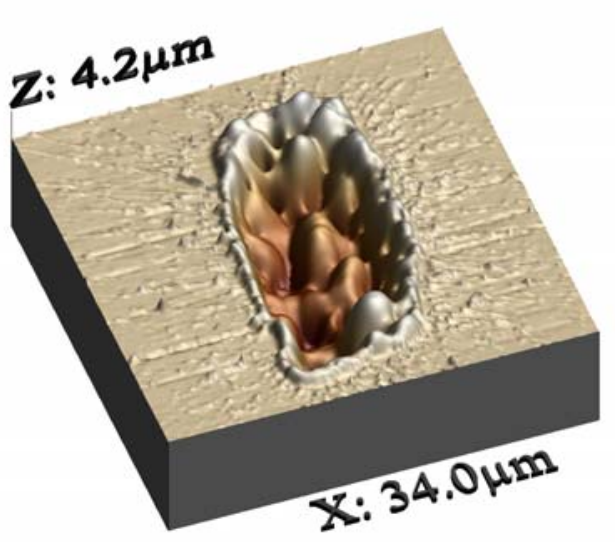

Figure 2: PMMA ablated by LCLS tuned at $1.5 \mathrm{~nm}$.

Attenuation length of X-rays becomes much shorter in materials like lead tungstate $\left(\mathrm{PbWO}_{4}\right.$; frequently used as a scintillator at HEP facilities [14]) because of its high mean atomic number $(\mathrm{Pb}, \mathrm{W})$ and high density $\left(=8.3 \mathrm{~g} / \mathrm{cm}^{3}\right)$ resulting in a stronger interaction with short-wavelength radiation. Comparable attenuation length we may get in lead iodide $\left(\mathrm{PbI}_{2}\right)$ which is often utilized as a semiconductor detector of ionizing radiation [15]. In this contribution, we summarize initial results of experiments carried out at the LCLS facility with the focused X-ray laser beam to determine the single-shot damage thresholds in two lead-containing monocrystalline materials, i.e., lead tungstate and lead iodide. For $\mathrm{PbWO}_{4}$, the results will be compared to the ablation data obtained in the XUV spectral region at the FLASH facility in Hamburg. 


\section{EXPERIMENTAL}

The experiment was carried out using the SXR experimental station [16] of the LCLS facility [5,6]. The SASE ( Amplified Spontaneous Emission) FEL X-ray source was operated with the electron bunch charge $250 \mathrm{pC}$ and 13 undulator segments tunable to deliver X-ray photons with energies between $800 \mathrm{eV}$ and $2000 \mathrm{eV}$ ( $\lambda$ from $1.55 \mathrm{~nm}$ to $0.62 \mathrm{~nm}$ ). Under these conditions, LCLS is expected to be working in its saturation regime. The duration of a single pulse was about $100 \mathrm{fs}$. An average pulse energy was of about $1 \mathrm{~mJ}$ in an unattenuated beam. Gas attenuator was used to adjust a fluence level at the sample surface. Pulse energy was determined for each shot by nitrogen-fluorescence detector. The transmission of the beamline was measured by using a photo-ionization Gas Monitor Detector (GMD) filled with a suitable rare gas. The LCLS beam was focused on the sample by Kirkpatrick-Baez mirrors with a focal length of $1.2 \mathrm{~m}$ [17]. The focusing system guaranties beam diameter at the sample surface of several micrometers in the tight focus. Ablation phenomena were studied changing sample position along the focused beam and varying pulse energy doing z-scans and F-scans, respectively. Proper sample positioning and focused beam characterization were secured with help of ablation imprint techniques [18]. After each shot fired on the sample surface, the target was moved to an unexposed position. Each sample was positioned perpendicularly to the incident LCLS beam in its tight focus.

Single crystals of lead tungstate have been grown by Czochralski technique at the Institute of Physics in Prague. $\mathrm{PbI}_{2}$ was prepared by direct synthesis from lead and iodine at the Prague Institute of Chemical Technology. The material was further purified by zone melting and grown by Bridgman-Stockbarger method [19]. Irradiated surfaces of both materials were investigated using Nomarski (DIC - differential interference contrast) optical microscope (BX51M DIC microscope, Olympus; Japan) and by an AFM microscope working in tapping mode (D3100 NanoScope Dimension controlled by NanoScope IV Control Station, Veeco; USA).

\section{RESULTS AND DISCUSSION}

DIC image of a typical crater created by the focused LCLS beam in $\mathrm{PbWO}_{4}$ above the ablation threshold can be seen in Fig. 3. Athough in the center of the damage pattern a circular region can be indicated with traces of surface melting, outer ablation contour is clean and sharp. Both the focal spot area and single-shot ablation threshold were determined from the plot (Fig. 4) of damaged surface areas as a function of a pulse energy logarithm, i.e, by Liu's technique. Details of the method may be found in refs. $[9,10,20]$. The focal spot area and the ablation threshold inferred from the data in Fig. 4 are $A_{\text {eff }}=(229 \pm 6) \mu \mathrm{m}^{2}$ and $\mathrm{F}_{\text {th }}=(0.11 \pm 0.03) \mathrm{J} / \mathrm{cm}^{2}$, respectively.

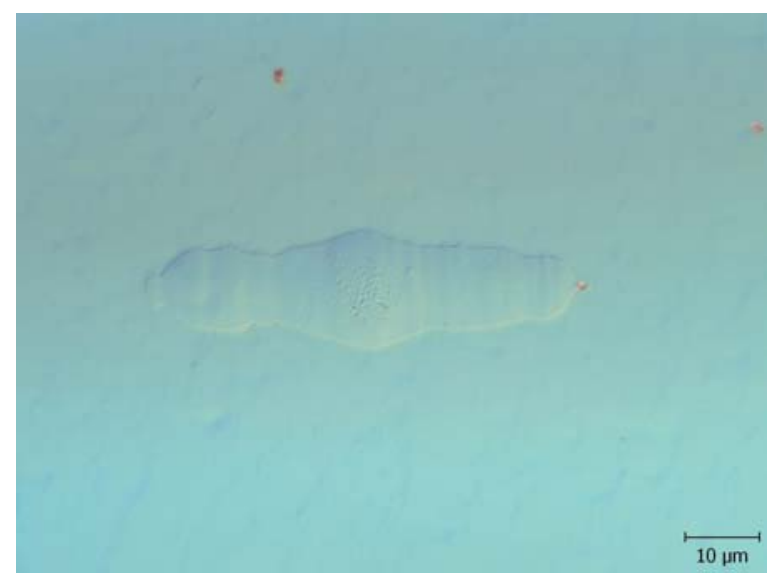

Figure 3: The DIC image of a crater formed in $\mathrm{PbWO}_{4}$ by the focused LCLS beam near the ablation threshold.

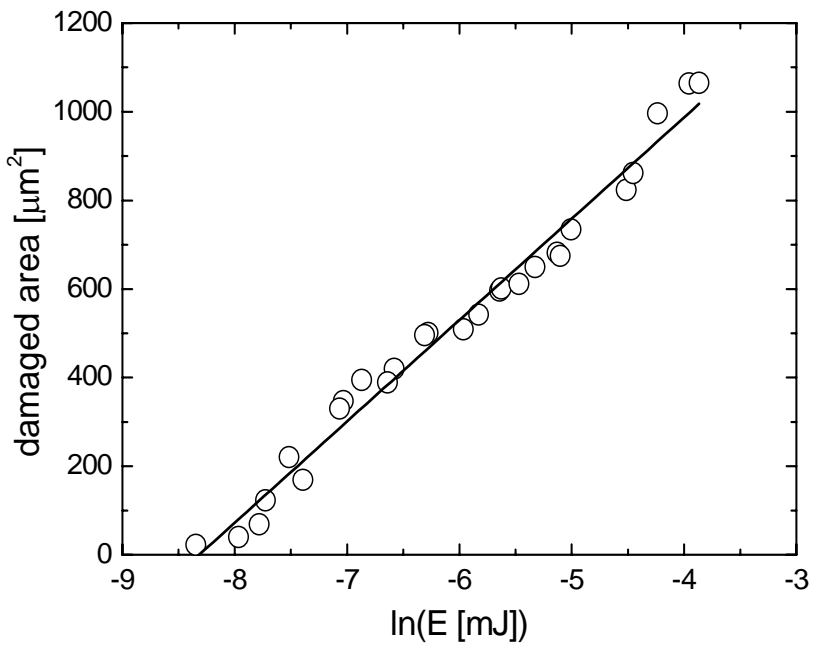

Figure 4: The dependence of $\mathrm{PbWO}_{4}$ surface area damaged by $800-\mathrm{eV}$ LCLS radiation on the pulse energy. 
The ablation threshold values obtained for $\mathrm{PbWO}_{4}$ irradiated by a single ultra-short pulse of XUV (FLASH) and X-ray (LCLS) laser radiation at different wavelengths are summarized in Tab. 1. For X-rays, the ablation threshold does not seem to be strongly dependent on a photon energy although an attenuation length listed from Henke's tables [21] changes a lot with changing wavelength. All the thresholds are slightly above $0.1 \mathrm{~J} / \mathrm{cm}^{2}$ from $800 \mathrm{eV}$ to $2 \mathrm{keV}$. For photons with an energy of $800 \mathrm{eV}$, the first ablative damage appears around $110 \mathrm{~mJ} / \mathrm{cm}^{2}$. This is a much lower value than expected taking into account the threshold obtained in the extreme ultraviolet spectral region at $13.5 \mathrm{~nm}$, i.e., $79 \mathrm{~mJ} / \mathrm{cm}^{2}$, and the ratio of attenuation lenths

Table 1: Single-shot damage thresholds in lead tungstate irradiated by free-electron lasers tuned at various wavelengths in XUV and $\mathrm{X}$-ray spectral regions.

\begin{tabular}{|c|c|c|c|c|c|}
\hline $\begin{array}{c}\text { wavelength } \\
{[\mathrm{nm}]}\end{array}$ & $\begin{array}{c}\text { photon energy } \\
{[\mathrm{eV}]}\end{array}$ & $\begin{array}{c}\text { atten. length } \\
\text { Henke }[\mathrm{nm}]\end{array}$ & $\begin{array}{c}\text { atten. length } \\
{[\mathrm{nm}]}\end{array}$ & $\begin{array}{c}\text { threshold fluence } \\
{\left[\mathrm{mJ} / \mathrm{cm}^{2}\right]}\end{array}$ & evaluation method \\
\hline 13.5 & 91.7 & 33.4 & - & 79 & measured: F-scan \\
\hline 1.55 & 800 & 170 & - & 400 & extrapolated from $13.5 \mathrm{~nm}$ \\
\hline 1.55 & 800 & 170 & 380 & 110 & measured: F-scan \\
\hline 0.77 & 1600 & 675 & - & 104 & measured: $z$-scan \\
\hline 0.62 & 2000 & 509 & - & $\sim 1200$ & extrapolated from $13.5 \mathrm{~nm}$ \\
\hline 0.62 & 2000 & 509 & - & $\sim 150$ & measured: F-scan \\
\hline
\end{tabular}

at these two wavelenths, i.e., $1.55 \mathrm{~nm}$ and $13.5 \mathrm{~nm}$. According to Henke's tables [21], the expected value should be around $400 \mathrm{~mJ} / \mathrm{cm}^{2}$ to reach at the wavelength of $1.55 \mathrm{~nm}$ a surface energy density corresponding to the threshold obtained at $13.5 \mathrm{~nm}$. Since the threshold is in principle given by surface energy density rate, the expected value should be at LCLS even higher because a LCLS pulse duration ranges around 100 fs while FLASH provides pulses of 30 fs. Moreover, the effective attenuation length determined from LCLS ablation data seems to be longer than a value listed in Henke's tables. An effective attenuation length of $380 \mathrm{~nm}$ was found for $800-\mathrm{eV}$ photons, while Henke [21] gives 170 $\mathrm{nm}$. This is another factor that could cause further decrease of estimated threshold value.

The effect is even more remarkable at a wavelength of $0.62 \mathrm{~nm}$, which is the shortest wavelength achievable at the SXR experimental station. The wavelength scaling provides a threshold estimate around $1.2 \mathrm{~J} / \mathrm{cm}^{2}$ while actually obtained value is by an order of magnitude lower, i.e., $0.15 \mathrm{~J} / \mathrm{cm}^{2}$. This finding can be considered as an evidence of the nonthermal character of X-ray ablation in this case.
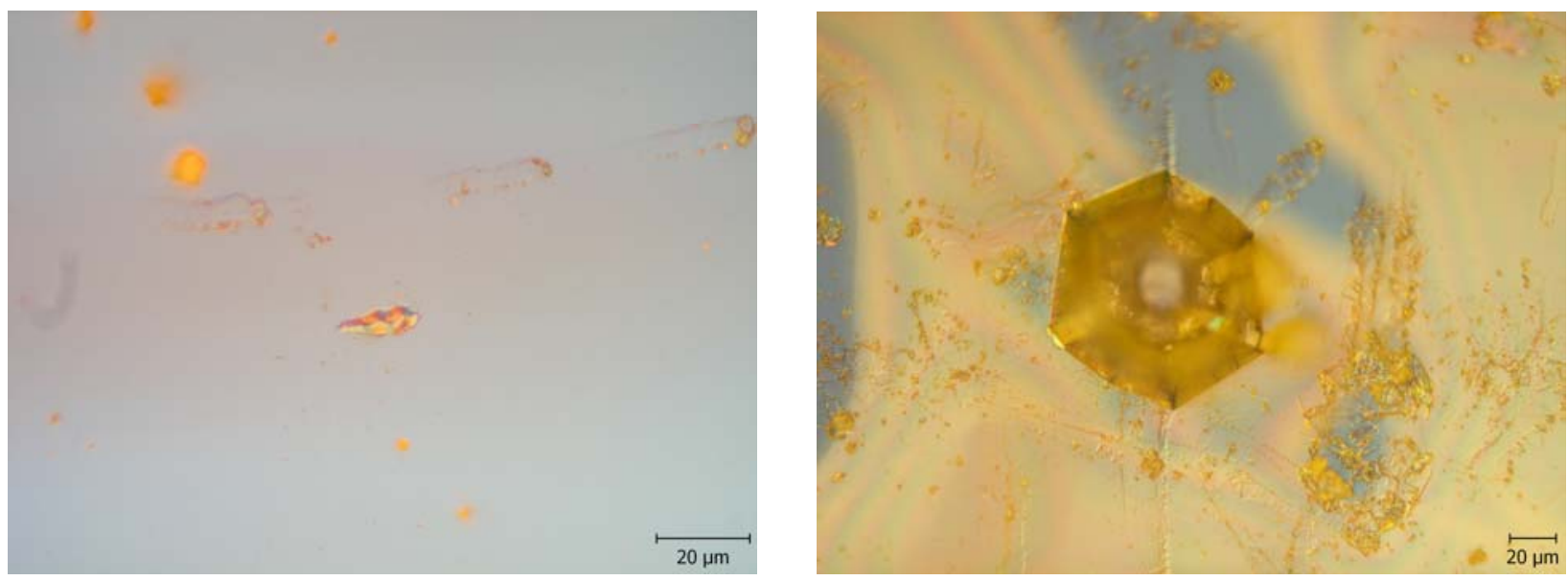

Figure 5: DIC images of $\mathrm{PbI}_{2}$ irradiated (left) near and (right) above the damage threshold by 800 -eV LCLS radiation. 
Monocrystalline $\mathrm{PbI}_{2}$ exhibits a single-shot ablation threshold around $4 \mathrm{~mJ} / \mathrm{cm}^{2}$ when irradiated by $1.5-\mathrm{nm}$ LCLS radiation (Fig. 5). Although there are wide error bars, i.e., $(-2 ;+32) \mathrm{mJ} / \mathrm{cm}^{2}$, the threshold of $\mathrm{PbI}_{2}$ is clearly much lower than that obtained in $\mathrm{PbWO}_{4}$ at the same LCLS wavelength. An attenuation length of $1.55-\mathrm{nm}$ radiation is according to Henke [21] quite the same in both materials, i.e., $170 \mathrm{~nm}$ and $166 \mathrm{~nm}$ for $\mathrm{PbWO}_{4}$ and $\mathrm{PbI}_{2}$, respectively. The reason is that the mean atomic number of lead iodide is greater than of tungstate but iodide density is slightly lower $\left(=6.1 \mathrm{~g} / \mathrm{cm}^{3}\right)$. $\mathrm{PbI}_{2}$ has lower thermal and radiation resistance than $\mathrm{PbWO}_{4}$. Melting points of $\mathrm{PbWO}_{4}$ and $\mathrm{PbI}_{2}$ are $1398 \mathrm{~K}$ [14] and 685 $\mathrm{K}$ [22], respectively. $\mathrm{PbI}_{2}$ [23-25] seems to be less radiation stable than $\mathrm{PbWO}_{4}$ [26,27] under comparable irradiation conditions. Experiments with conventional sources of ionizing radiation are in progress to compare $\mathrm{PbI}_{2}$ and $\mathrm{PbWO}_{4}$ radiation resistance at the same irradiation conditions. Mechanical damage to lead iodide single crystal appears at high fluences. In Fig. 5 on the right, a hexagonal pattern is broken out the layered structure of $\mathrm{PbI}_{2}$ because of a high strain rate in the near surface layer. The shape of the pattern originates from the hexagonal symmetry of $\mathrm{PbI}_{2}$ crystal lattice (i.e., layered structure of $C 6$ type - $\mathrm{CdI}_{2}$ ). The nature of mechanical-like damage to solids irradiated by a single LCLS pulse was recently discussed in ref. [28] for low-Z materials.

\section{CONCLUSIONS}

The dependence of an area of $\mathrm{PbWO}_{4}$ surface damaged by a single shot of $1.55-\mathrm{nm}(800 \mathrm{eV})$ laser radiation on pulse energy shows clearly that ablation of this material by a single laser shot begins at $F_{\text {th }}=110 \mathrm{~mJ} / \mathrm{cm}^{2}$. However, wavelength scaling of FLASH results gives an expected value of $F_{t h}=400 \mathrm{~mJ} / \mathrm{cm}^{2}$. At $0.62 \mathrm{~nm}$, the difference between the value experimentally determined at the LCLS facility and the value estimated by scaling attenuation lengths is even greater. It indicates a key role of non-thermal, photon energy dependent processes in the X-ray ablation. Observed behavior cannot be explained only by the wavelength dependence of energy density rate on the sample surface and in the near-surface region. LCLS-irradiated $\mathrm{PbI}_{2}$ exhibits much lower single-shot ablation threshold than $\mathrm{PbWO}_{4}$. This is not surprising because $\mathrm{PbI}_{2}$ is thermally and radiation less resistant than $\mathrm{PbWO}_{4}$.

\section{ACKNOWLEDGEMENTS}

This work was partially funded by the Czech Ministry of Education (Projects LC510, LC528, LA08024, ME10046), Czech Science Foundation (Grants P108/11/1312 and P205/11/0571) and by Academy of Sciences of the Czech Republic (Grants Z10100523, IAA400100701, and KAN300100702). The research leading to the results presented here has received funding from EC's 6th FP under grant agreement No. RII3-CT-2003-506350. This work performed under the auspices of the U.S. Department of Energy by Lawrence Livermore National Laboratory under Contract DE-AC5207NA27344. Portions of this research were carried out on the SXR Instrument at the Linac Coherent Light Source (LCLS), a division of SLAC National Accelerator Laboratory and an Office of Science user facility operated by Stanford University for the U.S. Department of Energy. The SXR Instrument is funded by a consortium whose membership includes the LCLS, Stanford University through the Stanford Institute for Materials Energy Sciences (SIMES), Lawrence Berkeley National Laboratory (LBNL), University of Hamburg through the BMBF priority program FSP 301, and the Center for Free Electron Laser Science (CFEL).

\section{REFERENCES}

[1] H. Wabnitz et al., "Multiple ionization of atom clusters by intense soft X-rays from a free-electron laser", Nature 420, 482 (2002).

[2] H. N. Chapman et al., "Femtosecond diffractive imaging with a soft-X-ray free-electron laser", Nature Phys. 2, 839 (2006).

[3] B. Nagler et al., "Turning solid aluminium transparent by intense soft X-ray photoionization", Nature Phys. 5, 693 (2009).

[4] E. L. Saldin, E. A. Schneidmiller, and M. V. Yurkov, The Physics of Free Electron Lasers, Springer-Verlag, BerlinHeidelberg-New York (2000). 
[5] H. Winick et al., "Short wavelength FELs using the SLAC linac", Nucl. Instrum. Meth. Phys. Res. A347, 199 (1994).

[6] P. Emma et al., "First lasing and operation of an ångstrom-wavelength free-electron laser", Nature Phot. 4, 641 (2010).

[7] M. Yabashi, A. Higashiya, K. Tamasaku, H. Kimura, T. Kudo, H. Ohashi, S. Takahashi, S. Goto, and T. Ishikawa, "Optics development for Japanese XFEL project", Proc. SPIE 6586, 658605 (2007).

[8] T. Tschentscher, H. Sinn, K. Tiedtke, and H. Wabnitz, "X-ray optics power considerations for high intensity x-ray free-electron lasers based on superconducting technology", Proc. SPIE 6586, 65860R (2007).

[9] J. Chalupský, L. Juha, J. Kuba, V. Hájková, J. Cihelka, P. Homer, M. Kozlová, T. Mocek, J. Polan, B. Rus, J. Krzywinsky, R. Sobierajski, H. Wabnitz, J. Feldhaus, and K. Tiedtke, "Utilizing ablation of solids to characterize a focused soft x-ray laser beam," Proc. SPIE 6586, 65860S-1 (2007) .

[10] J. Chalupský et al., "Characteristics of focused soft X-ray free-electron laser beam determined by ablation of organic molecular solids," Opt. Express 15, 6036 (2007) .

[11] J. Chalupský, J. Krzywinski, L. Juha, V. Hájková, J. Cihelka, T. Burian, L. Vyšín, J. Gaudin, A. Gleeson, M. Jurek, A. R. Khorsand, D. Klinger, H. Wabnitz, R. Sobierajski, M. Störmer, K. Tiedtke, and S. Toleikis, "Spot size characterization of focused non-Gaussian X-ray laser beams", Opt. Express 18, 27836 (2010)

[12] K. Tiedtke et al., "The soft x-ray free-electron laser FLASH at DESY: beamlines, diagnostics and end-stations," New J. Phys. 11, 023029 (2009)

[13] A. J. Nelson et al.: "Soft x-ray free electron laser microfocus for exploring matter under extreme conditions", Opt. Express 17, 18271 (2009)

[14] A. A. Annenkov, M. V. Korzhik, and P. Lecoq: "Lead tungstate scintillation material", Nucl. Instrum. Meth. Phys. Res. A 490, 30 (2002).

[15] J. C. Lund, F. Olschner, and A. Burger: "Lead iodide crystals and detectors", Semicond. Semimet. 43, 443 (1995).

[16] S. Moeller et al., "Photon beamlines and diagnostics at LCLS", Nucl. Instrum. Meth. Phys. Res. A 635, S6 (2011).

[17] A. Barty, R. Soufli, T. McCarville, S. L. Baker, M. J. Pivovaroff, P. Stefan, and R. Bionta, "Predicting the coherent X-ray wavefront focal properties at the Linac Coherent Light Source (LCLS) X-ray free electron laser", Opt. Express 17, 15508 (2009).

[18] J. Chalupský, P. Boháček, V. Hájková, S. P. Hau-Riege, P. A. Heimann, L. Juha, J. Krzywinski, M. Messerschmidt, S. P. Moeller, B. Nagler, M. Rowen, W. F. Schlotter, M. L. Swiggers, and J. J. Turner, "Comparing different approaches to characterization of focused x-ray laser beam", Nucl. Instrum. Meth. Phys. Res. A 631, 130 (2011).

[19] M. Matuchova, K. Zdansky, J. Zavadil, A. Danilewsky, J. Maixner, and D. Alexiev, "Electrical, optical and structural properties of lead iodide", J. Mater. Sci. Mater. Electron. 20, 289 (2009).

[20] J. M. Liu, "Simple technique for measurements of pulsed Gaussian-beam spot sizes," Opt. Lett. 7, 196 (1982).

[21] http://henke.lbl.gov/optical_constants/atten2.html

[22] R. F. Rolsten, Iodide Metals and Metal Iodides, Wiley, NY-London (1961).

[23] M. R. Tubbs, "The optical properties and decomposition of halides with layer structures I and II", phys. stat. sol. $b$ 49, 11 (1972) and 67, 11 (1975) and references cited therein.

[24] A. J. Forty, "Observations of the decomposition of crystals of lead iodide in the electron microscope", Phil. Mag. 5, 787 (1960).

[25] A. J. Forty, "The precipitation of lead during decomposition of lead iodide by electron irradiation", Phil. Mag. 6, 895 (1961).

[26] M. Kobayashi, M. Ishiib, and Y. Usuki, "Comparison of radiation damage in different $\mathrm{PbWO}_{4}$ scintillating crystals", Nucl. Instrum. Meth. Phys. Res. A 406, 442 (1998). 
[27] M. Nikl, E. Mihokova, V. Laguta, J. Pejchal, S. Baccaro, and A. Vedda, "Radiation damage processes in complexoxide scintillators", Proc. SPIE 6586, 65860E (2007) and references cited therein.

[28] S. P. Hau-Riege et al., "Interaction of short x-ray pulses with low-Z x-ray optics materials at the LCLS free-electron laser", Opt. Express 18, 23933 (2010). 